\title{
THE NORMAL CURVATURE OF TOTALLY REAL SUBMANIFOLDS OF $S^{6}(1)$
}

\author{
by P. J. DE SMET, F. DILLEN, L. VERSTRAELEN and L. VRANCKEN ${ }^{\dagger}$
}

(Received 30 September, 1996)

\begin{abstract}
We prove the pointwise inequality $0 \geq \rho+\rho^{\perp}-1$ involving the normalized scalar curvature $\rho$ and normal scalar curvature $\rho^{\perp}$ of a totally real 3-dimensional submanifold of the nearly Kaehler 6-sphere. Further we classify submanifolds realizing the equality in this inequality.
\end{abstract}

1. Introduction. Let $M^{n}$ be an $n$-dimensional (immersed) submanifold of an $m$-dimensional real is space form $N^{m}(c)$. If $R^{\perp}$ is the curvature tensor of the normal connection, $\left\{e_{1}, \ldots, e_{n}\right\}$ an orthonormal basis of tangent vector fields and $\left\{\xi_{1}, \ldots, \xi_{m-n}\right\}$ an orthonormal basis of normal vector fields, then the (normalized) normal scalar curvature $\rho^{\perp}$ is defined in [7] by

$$
\rho^{\perp}=\frac{2}{n(n-1)}\left(\sum_{i<j=1}^{n} \sum_{r<s=1}^{(m-n)}\left\langle R^{\perp}\left(e_{i}, e_{j}\right) \xi_{r}, \xi_{s}\right\rangle^{2}\right)^{1 / 2} .
$$

For $n=2$, this definition is the same as the definition of "normal curvature", given in [11]. The following was conjectured in [7].

Conjecture. Let $\phi: M^{n} \rightarrow N^{m}(c)$ be an isometric immersion. Then at every point $p$, we have

$$
|H|^{2} \geq \rho+\rho^{\perp}-c
$$

where $\rho$ is the normalized scalar curvature, and $H$ is the mean curvature vector of $M^{n}$.

This conjecture was proved for $n=2, m=4$ and $c=0$ by Wintgen [13]; for $n=2$ and $m \geq 4$ by Guadalupe and Rodriguez [11]; for $n \geq 2$ and $m=n+2$ by the authors in [7]. For $n \geq 2$ and $m=m+1$, in which case $\rho^{\perp}$ is trivially zero, the conjecture follows from a more general result of Chen in [4] stating that for arbitrary submanifolds of real space forms, $|H|^{2} \geq \rho-c$.

In this paper, we prove the conjecture for 3-dimensional totally real submanifolds of $S^{6}$. Note that such submanifolds are always minimal [10]. In particular, we prove the following theorem.

tThe second and fourth authors are Senior Research Assistants of the National Fund and Scientific Research (Belgium). Research supported by the grant OT/TBA/95/9 of the Research Council of the Katholieke Universiteit Leuven.

Glasgow Math. J. 40 (1998) 199-204. 
THEOREM 1. Let $x: M^{3} \rightarrow S^{6}(1)$ be a totally real isometric immersion. Then

$$
0 \geq \rho+\rho^{\perp}-1
$$

If we define, following Chen [3], a Riemannian invariant $\delta_{M}$ by

$$
\delta_{M}(p)=\frac{n(n-1)}{2} \rho(p)-\inf K(p),
$$

where inf $K$ is the function assigning to each $p \in M$ the infimum of $K(\pi), \pi$ running over all planes in $T_{p} M$, then it is proved in [3] that any minimal submanifold of a unit sphere satisfies $\delta_{M} \leq \frac{1}{2}(n+1)(n-2)$. For totally real 3-dimensional submanifolds of $S^{6}(1)$, this becomes $\delta_{M} \leq 2$.

Theorem 2. Let $X: M^{3} \rightarrow S^{6}(1)$ be a totally real isometric immersion, and let $p \in M$. Then the following statements are equivalent.

(1) $\rho+\rho^{\perp}=1$ at $p$,

(2) $\delta_{M}=2$ at $p$,

(3) There exists an orthonormal basis $\left\{e_{1}, e_{2}, e_{3}\right\}$ at $p$ such that

$$
\begin{array}{lll}
h\left(e_{1}, e_{1}\right)=\lambda J e_{1} & h\left(e_{2}, e_{2}\right)=-\lambda J e_{1} & h\left(e_{1}, e_{2}\right)=-\lambda J e_{2}, \\
h\left(e_{2}, e_{3}\right)=0 & h\left(e_{1}, e_{3}\right)=0 & h\left(e_{3}, e_{3}\right)=0 .
\end{array}
$$

Totally real immersions with $\delta_{M}=2$ at every point are completely classified, see [5], [6] and [9]. The classification is summarized in the following theorems from [9].

Theorem 3. Let $\phi: N_{1} \rightarrow C P^{2}(4)$ be a holomorphic curve in $C P^{2}(4)$. Let $P N_{1}$ be the circle bundle over $N_{1}$ induced by the Hopf fibration $p: S^{5}(1) \rightarrow C P^{2}(4)$ and let $\psi$ be the isometric immersion such that the following diagram commutes.

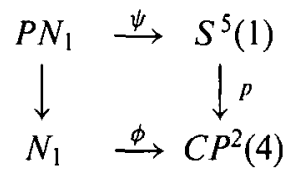

Then there exists a totally geodesic embedding $i$ of $S^{5}(1)$ into the nearly Kähler 6-sphere such that the immersion $i \circ \psi: P N_{1} \rightarrow S^{6}(1)$ is a 3-dimensional totally real immersion in $S^{6}(1)$ with $\delta_{P N_{1}}=2$. Conversely let $F: M^{3} \rightarrow S^{6}(1)$ be a totally real immersion which is in linearly full in $S^{6}(1)$. Then $M^{3}$ automatically satisfies $\delta_{M}=2$ and there exists a totally geodesic $S^{5}(1)$ and a holomorphic curve $\phi: N_{1} \rightarrow C P^{2}(4)$ such that $F$ is congruent to $\psi$, which is obtained from $\phi$ as above.

THEOREM 4. Let $\bar{\phi}: N_{2} \rightarrow S^{6}(1)$ be an almost complex curve (with second fundamental form $\alpha$ ) without totally geodesic points. Denote by $\mathrm{UN}_{2}$ the unit tangent bundle over $\mathrm{N}_{2}$ and define a map

$$
\bar{\psi}: U N_{2} \rightarrow S^{6}(1): v \mapsto \bar{\phi}_{*}(v) \times \frac{\alpha(v, v)}{\|\alpha(v, v)\|}
$$


Then $\bar{\psi}$ is a (possibly branched) totally real immersion into $S^{6}(1)$ satisfying $\delta_{U N_{2}}=2$. Moreover, the immersion is linearly full in $S^{6}(1)$. Conversely let $F: M^{3} \rightarrow S^{6}(1)$ be a linearly full totally real immersion of a 3-dimensional manifold satisfying $\delta_{M}=2$. Let $p$ be a non totally geodesic point of $M^{3}$. Then there exists a (possibly branched) almost complex curve $\bar{\phi}: N_{2} \rightarrow S^{6}(1)$ such that, around $p, F$ is congruent to $\bar{\psi}$, which is obtained from $\bar{\phi}$ as above.

So the following corollary follows immediately from the previous theorems.

COROLlary 1. If $f: M^{3} \rightarrow S^{6}(1)$ is a totally real isometric immersion, satisfying $\rho+\rho^{\perp}=1$, then $f$ is one of the immersions given in Theorem 3 or Theorem 4 above.

2. The nearly Kaehler structure on $S^{6}$. We briefly describe how the standard nearly Kähler structure on $S^{6}(1)$ arises in a natural manner from Cayley multiplication. The multiplication on the Cayley numbers $\mathcal{O}$ may be used to define a vector cross product $x$ on the purely imaginary Cayley numbers $R^{7}$. The standard nearly Kähler structure on $S^{6}(1) \subset R^{7}$ is then obtained as follows.

$$
J u=x \times u, \quad u \in T_{x} S^{6}(1), x \in S^{6}(1) .
$$

Then $J$ is an orthogonal almost complex structure on $S^{6}(1)$. In fact $J$ is a nearly Kähler structure in the sense that the (2,1)-tensor field $G$ on $S^{6}(1)$, defined by $G(X, Y)=\left(\widetilde{\nabla}_{X} J\right)(Y)$, where $\widetilde{\nabla}$ is the Levi-Civita connection on $S^{6}(1)$, is skew-symmetric. For more information on the properties of $\times, J$ and $G$, we refer to [2], [1] and [8].

3. Totally real immersions with $\delta_{M}=2$. An immersion $F: M^{3} \rightarrow S^{6}(1)$ is called totally real if the almost complex structure $J$ maps the tangent space into the normal space. In [10] Ejiri proved that a 3-dimensional totally real submanifold of $S^{6}(1)$ is orientable and minimal and that $G(X, Y)$ is orthogonal to $M$, for tangent vectors $X$ and $Y$. We denote that LeviCivita connection of $M$ by $\nabla$. The formulas of Gauss and Weingarten are then respectively given by

$$
\begin{gathered}
\widetilde{\nabla}_{X} F_{*} Y=F_{*}\left(\nabla_{X} Y\right)+h(X, Y), \\
\widetilde{\nabla}_{X} \eta=-F_{*}\left(A_{\eta} X\right)+\nabla_{X}^{\perp} \eta,
\end{gathered}
$$

for tangent vector fields $X$ and $Y$ and normal vector field $\eta$. The second fundamental form $h$ is related to $A_{\eta}$ by $\langle h(X, Y), \eta\rangle=\left\langle A_{\eta} X, Y\right\rangle$. From (3.1) and (3.2), we find that

$$
\begin{gathered}
\nabla_{X}^{\perp} J F_{*}(Y)=J F_{*}\left(\nabla_{X} Y\right)+G\left(F_{*} X, F_{*} Y\right), \\
F_{*}\left(A_{J Y} X\right)=-J h(X, Y) .
\end{gathered}
$$

The above formulas immediately imply that $\left\langle h(X, Y\rangle, J F_{*} Z\right\rangle$ is totally symmetric. 
The fundamental equations of Gauss, Codazzi and Ricci then respectively state that

$$
\begin{aligned}
&\langle R(X, Y) Z, W\rangle=\langle Y, Z\rangle\langle X, W\rangle-\langle X, Z\rangle\langle Y, W\rangle \\
&+\langle h(Y, Z), h(X, W)\rangle-\langle h(X, Z), h(Y, W)\rangle, \\
&(\nabla h)(X, Y, Z)=(\nabla h)(Y, X, Z), \\
&\left.\left\langle R^{\perp}(X, Y) \xi, \eta\right\rangle=\left\langle\left[A_{\xi}, A_{\eta}\right] X, Y\right\rangle\right],
\end{aligned}
$$

where $X, Y, Z, W$ are tangent vector fields and $\xi$ and $\eta$ are normal vector fields. From these equations, one obtains easily that

$$
\left\langle R^{\perp}(X, Y) J Z, J W\right\rangle=\langle R(X, Y) Z, W\rangle-\langle Y, Z\rangle\langle X, W\rangle+\langle X, Z\rangle\langle Y, W\rangle .
$$

4. Proofs. Let $F: M^{3} \rightarrow S^{6}$ be a totally real immersion. We identify $M^{3}$ with its image in $S^{6}$. Let $p \in M$ and assume that $p$ is not a totally geodesic point. Following [10], see also [12], there exists an orthonormal basis $\left\{e_{1}, e_{2}, e_{3}\right\}$ at the point $p$ such that $G\left(e_{1}, e_{2}\right)=$ $J_{e_{3}}, G\left(e_{2}, e_{3}\right)=J e_{1}, G\left(e_{3}, e_{1}\right)=J_{e_{2}}$, and

$$
\begin{array}{ll}
h\left(e_{1}, e_{1}\right)=(a+b) J e_{1}, & h\left(e_{2}, e_{2}\right)=-a J e_{1}+c J e_{2}-d J e_{3}, \\
h\left(e_{1}, e_{2}\right)=-a J e_{2}, & h\left(e_{2}, e_{3}\right)=-d J e_{2}-c J e_{3}, \\
h\left(e_{1}, e_{3}\right)=-b J e_{3}, & h\left(e_{3}, e_{3}\right)=-b J e_{1}-c J e_{2}+d J e_{3},
\end{array}
$$

where $a+b>0$. A straightforward computation using the Gauss equation gives that

$$
\begin{aligned}
& K\left(e_{1} \wedge e_{2}\right)=1-2 a^{2}-a b, \\
& K\left(e_{1} \wedge e_{3}\right)=1-2 b^{2}-a b, \\
& K\left(e_{2} \wedge e_{3}\right)=1+a b-2\left(c^{2}+d^{2}\right) .
\end{aligned}
$$

Hence we obtain that

$$
3 \rho=\sum_{i<j} K\left(e_{i} \wedge e_{j}\right)=3-\left(2 a^{2}+2 b^{2}+2 c^{2}+2 d^{2}+a b\right) .
$$

Since $a+b>0$, we have $-2 a b<a^{2}+b^{2}$ and from the Ricci equation we find

$$
\begin{aligned}
9\left(\rho^{\perp}\right)^{2}= & \frac{1}{2} \sum_{r<s}\left\|\left[A_{J e_{r}}, A_{J e_{s}}\right]\right\|^{2} \\
= & 2\left(c^{2}+d^{2}\right)(a-b)^{2}+a^{2}(2 a+b)^{2}+b^{2}(a+2 b)^{2}+\left(a b-2\left(c^{2}+d^{2}\right)\right)^{2} \\
= & 4 a^{4}+4 b^{4}+4 c^{4}+4 d^{4}+a^{2} b^{2}+2 a^{2} c^{2}-8 a b c^{2}+2 b^{2} c^{2} \\
& \quad+2 a^{2} d^{2}-8 a b d^{2}+2 b^{2} d^{2}+4 a^{3} b+2 a^{2} b^{2}+4 a b^{3}+8 c^{2} d^{2} \\
= & 4 a^{4}+4 b^{4}+4 c^{4}+4 d^{4}+a^{2} b^{2}+2 a^{2} c^{2}-12 a b c^{2}+2 b^{2} c^{2} \\
& +2 a^{2} d^{2}-12 a b d^{2}+2 b^{2} d^{2}+4 a^{3} b+2 a^{2} b^{2}+4 a b^{3}+8 c^{2} d^{2} \\
& +4 a b c^{2}+4 a b d^{2}
\end{aligned}
$$




$$
\begin{aligned}
\leq & 4 a^{4}+4 b^{4}+4 c^{4}+4 d^{4}+a^{2} b^{2}+2 a^{2} c^{2}+6\left(a^{2}+b^{2}\right) c^{2}+2 b^{2} c^{2} \\
& +2 a^{2} d^{2}+6\left(a^{2}+b^{2}\right) d^{2}+2 b^{2} d^{2}+4 a^{3} b+2 a^{2} b^{2}+4 a b^{3}+8 c^{2} d^{2} \\
& +4 a b c^{2}+4 a b d^{2} \\
= & 4 a^{4}+4 b^{4}+4 c^{4}+4 d^{4}+a^{2} b^{2}+2 a^{2} b^{2}+8 a^{2} c^{2}+8 b^{2} c^{2} \\
& +8 a^{2} d^{2}+8 b^{2} d^{2}+4 a^{3} b+4 a b^{3}+8 c^{2} d^{2}+4 a b c^{2}+4 a b d^{2} \\
\leq & 4 a^{4}+4 b^{4}+4 c^{4}+4 d^{4}+a^{2} b^{2}+8 a^{2} b^{2}+8 a^{2} c^{2}+8 a^{2} d^{2} \\
& +4 a^{3} b+8 b^{2} c^{2}+8 b^{2} d^{2}+4 a b^{3}+8 c^{2} d^{2}+4 a b c^{2}+4 a b d^{2} \\
= & \left(2 a^{2}+2 b^{2}+2 c^{2}+2 d^{2}+a b\right)^{2} .
\end{aligned}
$$

Since $2 a^{2}+2 b^{2}+a b \geq 0$, we deduce from this that

$$
3 \rho^{\perp} \leq 2 a^{2}+2 b^{2}+2 c^{2}+2 d^{2}+a b .
$$

Hence, using (4.1), this becomes

$$
3 \rho^{\perp} \leq 3-3 \rho
$$

which proves the inequality. This finishes the proof of Theorem 1.

Let us assume now that the equality is realized in (1.1) at the point $p$. Equality in (4.2) implies that $c=d=0$ and equality in (4.3) that $a b=0$. So, if necessary, by replacing $e_{2}$ and $e_{3}$ by $-e_{3}$ and $e_{2}$, we may assume that $a \neq 0$ and $b=0$. This proves that (1) and (3) of Theorem 2 are equivalent. The equivalence of (2) and (3) is proved in [5]. This completes the proof of Theorem 2 .

REMARK 1. Using (3.5), one can always express the normal scalar curvature in terms of intrinsic invariants. After a straightforward computation, we obtain that

$$
3\left(\rho^{\perp}\right)^{2}=\frac{1}{12}\|R\|^{2}-2 \rho+1 .
$$

So (1.1) is in fact an intrinsic obstruction.

\section{REFERENCES}

1. J. Bolton, L. Vrancken and L. M. Woodward, On almost complex curves in the nearly Kähler 6-sphere, Quart. J. Math. Oxford Ser. (2) 45 (1994), 407-427.

2. E. Calabi, Construction and properties of some 6-dimensional almost complex manifolds, Trans. Amer. Math. Soc. 87 (1958), 407-438.

3. B. Y. Chen, Some pinching and classification theorems for minimal submanifolds, Arch Math. (Basel) 60 (1993), 568-578.

4. B. Y. Chen, Mean curvature and shape operator of isometric immersions in real-space-forms, Glasgow Math. J. 38 (1996), 87-97.

5. B.-Y. Chen, F. Dillen, L. Verstraelen and L. Vrancken, Two equivariant totally real immersions into the nearly Kähler 6-sphere and their characterization, Japanese J. Math. (N.S.) 21 (1995), 207-222. 
6. B. Y. Chen, F. Dillen, L. Verstraelen and L. Vrancken, Characterizing a class of totally real submanifolds of $S^{6}(1)$ by their sectional curvatures, Tôhoku Math. J. 47 (1995), 185-198.

7. P. J. De Smet, F. Dillen, L. Verstraelen and L. Vrancken, A pointwise inequality in submanifold theory (1996), Arch. Math. (Brno), to appear.

8. F. Dillen, L. Verstraelen and L. Vrancken, Classification of totally real 3-dimensional submanifolds of $S^{6}(1)$ with $K \geq \frac{1}{16}$, J. Math. Soc. Japan 42 (1990), 565-584.

9. F. Dillen and L. Vrancken, Totally real Submanifolds in $S^{6}$ satisfying Chen's Equality, Trans. Amer. Math. Soc. 348 (1996), 1633-1646.

10. N. Ejiri, Totally real submanifolds in a 6-sphere, Proc. Amer. Math. Soc. 83 (1981), 759-763.

11. I. V. Guadalupe and L. Rodriguez, Normal curvature of surfaces in space forms, Pacific $J$. Math. 106 (1983), 95-103.

12. L. Vrancken, Locally symmetric submanifolds of the nearly Kaehler $S^{6}$, Algebras, Groups and Geometries 5 (1988), 369-394.

13. P. Wintgen, Sur l'inégalité de Chen-Willmore, C. R. Acad. Sc. Paris 288 (1979), 993-995.

Katholieke Universiteit Leuven

DEPARTEMENT WISKUNDE

CelestijnenlaAn $200 \mathrm{~B}$

B-3001 LEUVEN

BELGIUM 